\title{
La internacionalización universitaria, un imperativo de la educación superior en el contexto latinoamericano actual
}

Dra. Rosario León Robaina

Universidad de Oriente. Cuba

rosariolr@uo.edu.cu, rosario.leon.

robaina@gmail.com

Dra. Luz Inmaculada Madera Soriano

Universidad Nacional por Educación

y Cultura. (República Dominicana)

imadera@yahoo.com

kelly.romero@cecar.edu.co
How to cite this article:

León Robaina, Rosario (2016); Madera Soriano, Inmaculada (2016); La internacionalización universitaria, un imperativo de la educación superior en el contexto latinoamericano actual. Revista Encuentros, Universidad Autónoma del Caribe, Vol. 14-02, pp pp.43- 59

DOI: http:/ / dx.doi.org/10.15665/ re.v14i2.779

\section{RESUMEN}

El artículo de investigación aborda el gran desafío de poder armonizar los intereses institucionales, nacionales e internacionales, a fin de poder cumplir con su misión social, de contribuir con la solución de los problemas sociales y promover el desarrollo humano, para lo cual ha de tomar en cuenta un contexto global. El resurgimiento de la internacionalización como realidad educativa es una consecuencia de esto. Su desarrollo se fundamenta en potenciar la voluntad, la capacidad y la identidad de la comunidad universitaria para la internacionalización. Teóricamente se partió de establecer los fundamentos para, metodológicamente desde un enfoque cualitativo, basado fundamentalmente en los métodos de análisis - síntesis, y el sistémico - estructural, establecer la estrategia de internacionalización universitaria. Los resultados del estudio evidencian que la estrategia propuesta es un instrumento que ha permitido potenciar el desarrollo de la cultural de la internacionalización, definir objetivos y estrategias que, desde una praxis, se materializan en los respectivos planes de acción, criterios de medida y grados de consecución.

Palabras clave: internacionalización universitaria, cooperación y colaboración internacional, desarrollo institucional. 


\title{
University internationalization. A higher education imperative in the Latin American context
}

\begin{abstract}
The challenge of being able to harmonize institutional, national and international interests, in order to fulfill the social mission contributing to solving social problems and promoting human development, for which a global context has to be taken into account in the present research. The resurgence of educational internationalization is actually a consequence of this. Its development is founded on the will to enhance the capacity and identity of the university community for internationalization. Theoretically it started setting up the foundations from a qualitative approach, methodologically based on structural and systemic action research to establish the university internationalization strategy. The results show that the proposed strategy is an instrument that has allowed enhancing the development of the internationalization culture, defining objectives and strategies, which from the praxis, are carried out in the respective action plans, measurement criteria, and levels of achievement.
\end{abstract}

Keywords: university internationalization, international cooperation and collaboration, institutional development.

\section{Universidade de internacionalização. Um imperativo de ensino superior na latin american atual contexto}

\begin{abstract}
RESUMO
Nas universidades no contexto atual enfrenta o desafio de ser capaz de harmonizar os interesses institucionais, nacionais e internacionais, a fim de cumprir sua missão social de contribuir para a solução dos problemas sociais e promover o desenvolvimento humano, para o qual deve ter em conta um contexto global. $\mathrm{O}$ ressurgimento da realidade internacionalização educativa é uma consequência desta. Seu desenvolvimento é baseado no fortalecimento da vontade, capacidade e identidade da comunidade universitária para a internacionalização. Teoricamente, dividida para estabelecer as bases teóricas para metodologicamente a partir da pesquisa-ação estrutural e sistémica, para estabelecer a estratégia de internacionalização da universidade. Os resultados mostram que a estratégia proposta é um instrumento que tem permitido melhorar o desenvolvimento da internacionalização cultural, definindo objectivos e estratégias a partir de uma práxis, são incorporados nos respectivos planos de acção, critérios de medição e níveis de realização.
\end{abstract}

Palavras-chave: internacionalização universitária, cooperação e colaboração internacional, o desenvolvimento institucional 


\section{Introducción}

Desde mediado del siglo XX el desarrollo social ha estado sujeto a las complejidades inherentes al mundo global, sus consecuencias y efectos inmediatos en la sociedad, la cultura y por ende, la educación superior se encuentra sometida a constantes cambios. En este contexto de importantes cambios globales, se ha redimensionado el concepto de lo humano y su desarrollo, el conocimiento y su interdisciplinaridad, el carácter universal, múltiple e interdependiente de la cultura, así como el rol de la educación como elemento fundamental para el desarrollo humano. El siglo XXI, por su parte, nace sujeto a las complejidades inherentes al mundo global, sus consecuencias y efectos inmediatos. En este escenario, la sociedad, la cultura y por ende, la educación superior, se encuentran sometidas a constantes cambios.

El fenómeno de la "globalización" ha ocupado todas las esferas de la vida humana y, en su perspectiva más amplia, puede definirse como "el flujo de tecnologías, economía, conocimiento, gente, valores e ideas a través de las fronteras" (Knight, J. y de Wit, H. 1997: 6). Se instala como modelo económico de fin del siglo $\mathrm{XX}$, ponderado por sus condiciones auspiciadoras del desarrollo humano. Sin embargo, en la actualidad se verifica un incremento sostenido en las demandas y necesidades sociales, brechas e inequidades, a escala mundial.

El análisis crítico de literatura y documentos relativos a la globalización ${ }^{7}$ y su impacto generalizado, permite concluir que los desafíos y trasformaciones sustantivas dadas a los distintos niveles del sistema social, incluido el ámbito educativo, presentan como impulsores clave una diversidad de factores, entre los que destaca: a) el desarrollo sostenido de los sistemas de información, la comunicación y la tecnología; b) el predominio de la economía de mercado, la liberación comercial, así como los nuevos espacios y modelos productivo-laborales; c) la reconfiguración demográfica del planeta, dada la creciente movilidad de personas a nivel internacional, así como las nuevas relaciones sociales y de género; d) una mayor interdependencia entre los pueblos y la búsqueda de la mundialización y homogeneización de la cultura en sus diversas manifestaciones; e) el enfoque en la Sociedad de la Información y el Conocimiento como elemento central de la dinámica económica, social y formativa en la sociedad actual; f) la revalorización del conocimiento como factor fundamental del desarrollo humano, lo que implica la necesidad un aprendizaje permanente; g) las nuevas relaciones entre los países, lo que ha generado la internacionalización de la justicia, la economía, la guerra y el terrorismo, el "mercado la información y el conocimiento" y de la educación; h) el compromiso de educar para la paz, la equidad, los derechos humanos y la preservación del medio ambiente; i) una mayor inversión privada y menos apoyo público para la educación.

Todo lo anterior ha provocado la necesidad de una educación superior que integre lo contextual, lo regional y lo internacional desde una mirada humana, de calidad, pertinente y accesible, que propicie la construcción de capacidades científicas que impulsen tanto la inserción en el mundo global, como el cambio social que se genera. Estos desafíos obligan a las universidades a realizar transformaciones y reorientar su cultura y su quehacer desde una perspectiva universal. (Madera, I. 2007). La internacionalización se constituye en la respuesta social, cultural y educativa al impacto de la globalización, a través de la cual el mundo académico pretende actuar de forma proactiva

Se han tomado en cuenta informes mundiales y regionales tales como Closingthe Gap in Education and Technology, del Banco Mundial (2002), Más allá de las fronteras, BID, (2002), Globalización y Desarrollo, CEPAL (2002),Informe Mundial de Desarrollo Humano, PNUD (2005); estudios sobre el impacto de la globalización en la educación superior como son el Informe Universidad Siglo XXI: Europa y América Latina. Regulación y Financiamiento, realizado por J. Bricall y J. Brunner para el CINDA (2000), El papel de las universidades en la Europa del Conocimiento, de la Comisión de las Comunidades Europeas (2003), así como las declaraciones de las cumbres mundiales de educación superior París '95 y' 98 ; el análisis crítico en torno a la globalización, representado a nivel mundial por autores entre los que destacan A. Gibbens (2000), J. Stiglitz (2002), G. de la Dehesa (2003)), los planteamientos de F. Sanz (2005) y de los radicales U. Beck (1998), Z. Bauman (1999), J. Petras (2000), N. Chomsky (2003), I. Wallerstein y C. Aguirre (2004), entre otros. 
salvaguardando desde la universalidad del conocimiento las particularidades locales.

Al compartir conocimientos, experiencias y recursos a través de los procesos de internacionalización, las universidades encuentran oportunidades para ampliar su visión y capacidades, redefinir su identidad, afirmar su autenticidad y actuar a partir de criterios de pertinencia, equidad y calidad, con impacto mundial. De este modo, la internacionalización se constituye en parte esencial de los procesos de desarrollo institucional universitario, a partir de la conformación de una cultura universitaria internacionalizada, que redimensione y adecue el proyecto universitario conforme al encargo de una sociedad global, plural y cambiante.

El salto cualitativo que ha de operarse en las instituciones de educación superior se hace viable en tanto la universidad, como sistema social complejo, tiene la capacidad de aprender, evolucionar y generar evolución de manera auto-organizada, en un diálogo inteligente con el entorno. Así, en respuesta a los procesos globales, las instituciones de nivel superior han desarrollado nuevas visiones y capacidades que les permitan desplazarse desde un paradigma educativo centrado en intereses nacionales, a uno contemplando la sociedad mundial, lo que ha sido denominado internacionalización de la educación superior. La misma, emerge como dimensión por excelencia con el potencial de impulsar y apoyar esos procesos de cambio institucional.

Al compartir conocimientos, experiencias y recursos a partir de procesos de internacionalización, las universidades encuentran oportunidades para ampliar su visión y capacidades, redefinir su identidad, afirmar su autenticidad y actuar a partir de criterios de pertinencia, equidad y calidad, con impacto mundial. Una transformación sustentable en el ámbito de la internacionalización, supone que la misma se constituya en parte esencial de los procesos de desarrollo institucional universitario, desde la conformación de una cultura universitaria internacionalizada, que redimensione y adecue el proyecto universitario conforme al encargo de una sociedad global, plural y cambiante.

En consecuencia, la internacionalización de la educación superior, sus mecanismos y paradigmas, pueden favorecer la transformación de las instituciones universitarias, conforme a la visión y dinámica propias de la sociedad contemporánea, la cual se articula en torno a la ciencia, la tecnología, la información y el conocimiento como generadores de bienestar económico y social. Sin em- bargo, en virtud de las dicotomías que caracterizan la era global, Latinoamérica continua transitando sus "vías" al desarrollo sin trascender efectivamente la periferia del mundo, sujeta a una realidad mundial incierta, compleja y contradictoria. Este rezago también encuentra expresión en la dinámica universitaria de la región, lo que incluye sus procesos de internacionalización. Ello ha quedado evidenciado en estudios realizados por instituciones locales y regionales, como es el conjunto de informes diagnósticos auspiciado en años recientes por el Instituto de Educación Superior para América Latina y el Caribe, IESALC-UNESCO ${ }^{8}$, entre otros.

Para actuar como elemento clave de la evolución social en el contexto actual, la universidad latinoamericana precisa cambiar, trascender los esquemas educativos tradicionales, las visiones fragmentadas, localistas y el frágil compromiso con el desarrollo humano, que limitan su relevancia. En este sentido, autores como A. Didriksson (2000), C. Tünnermann (2003) y J. Brunner (2002), entre otros, han referido la necesidad de cambios e innovaciones en la universidad latinoamericana, en respuesta a los nuevos compromisos que exige a la educación superior la sociedad actual. La internacionalización de la educación superior juega un papel fundamental en ese proceso, en es- 
pecial cuando es considerada como un eje transversal que favorece un desarrollo cultural universitario orientado por la apropiación de lo universal, enfoque abordado por investigadores tales como J. Knight y H. de Wit (1999), J. Gacel-Ávila (2000) y J. Sebastián (2000).

En consecuencia, la internacionalización de la educación superior, sus mecanismos y paradigmas deben favorecer la transformación de las instituciones universitarias, conforme a la visión y dinámica propias de la sociedad contemporánea, la cual se articula en torno a la ciencia, la tecnología, la información y el conocimiento como generadores de bienestar económico y social, de ahí la necesidad de proponer estrategias articuladas e integrales que permitan dar una respuesta social, cultural y educativa al impacto de la globalización, a través de la cual el mundo académico pretende actuar, de forma proactiva, salvaguardando las particularidades regionales y locales. He aquí el objetivo de la propuesta que se presenta.

La internacionalización constituye, por tanto, la respuesta social, cultural y educativa al impacto de la globalización, a través de la cual el mundo académico pretende actuar de forma proactiva ante este fenómeno, salvaguardando las particularidades locales. Además de referir a la presencia y visibilidad regional o mundial de las instituciones en pos de mayor influencia, calidad y eficacia, la internacionalización propicia la incorporación de la visión universal en su cultura y su dinámica. Favorece la armonización de lo local, lo regional y lo mundial en la praxis universitaria y promueve una globalización solidaria, de especial beneficio para los países periféricos.

El reconocimiento por la educación superior del papel de la internacionalización en cada uno de los procesos universitarios ha gestado un cambio en el actuar de la comunidad universitaria, constituyéndose esta en una de las dimensiones del desarrollo institucional. Corresponde por tanto a las universidades hacer de la internacionalización no un proceso más dentro de las estrategias institucionales, sino, convertirla en el eje transversal que marca el desarrollo y actuar universitario para su reconocimiento en cada uno de los espacios en los cuales ella se desarrolla: territorial, nacional, regional, mundial.

La consolidación de la internacionalización debe ser parte de la planificación estratégica universitaria y convertirse en un actuar permanente perfeccionándose y adecuándose a los cambios que el entorno nacional e internacional han demandado de la institución.

\section{Metodología}

Esta investigación es de tipo transversal con un enfoque cualitativo, basado fundamentalmente en los métodos teóricos de análisis
- síntesis, holístico - dialéctico y el sistémico - estructural - funcional, utilizados a lo largo de toda la investigación. De igual forma como métodos empíricos la observación y el análisis documental.

\section{II.1 Fundamentos teóricos}

La internacionalización constituye una gran fuerza para el desarrollo de una universidad moderna atenta al panorama que nos demanda el mundo de hoy. Está orientada para lograr la imagen deseada del profesor universitario, fundamentada en esquemas de formación: ante todo humanista, profesional de alto nivel, investigador, con gran sensibilidad social, lo cual sólo se puede alcanzar con el desarrollo de Instituciones de Educación Superior de mayor trascendencia académica, en el contexto internacional.

Para la mayoría de las Instituciones de Educación Superior de los países en vías de desarrollo, la incorporación de estrategias destinadas a desarrollar la cooperación y la internacionalización ha demostrado ser un factor catalizador de sus procesos de adecuación al entorno global, redimensionados conforme a criterios e indicadores de calidad propios de países desarrollados. ¿Cuáles principios deben regir la internacionalización de las universidades latinoamericanas?

- En primer, lugar se requiere desplazarse desde un paradigma educativo centrado tradicional- 
mente en intereses nacionales a uno contemplando la sociedad mundial.

"Parte esencial de las tareas de la educación superior es que debe vincularse estrechamente con su comunidad local, regional y nacional para, desde ese enraizamiento, abrirse al mundo con una visión universal para forjar "ciudadanos del mundo" capaces de comprometerse con la problemática global, de apreciar y valorar la diversidad cultural como fuente de enriquecimiento del patrimonio de la humanidad..." (Tuennermann, 1998).

- En segundo lugar, es importante comprender la estrategia de Internacionalización, en toda su dimensión. La Globalización se refiere al "flujo de conocimientos, tecnología, personas, valores, ideas que trascienden las fronteras...afecta a cada país de manera diferente, en relación con su historia, tradiciones, cultura y prioridades..." (Knight y de Wit, 1997). La internacionalización, por el contrario, describe una respuesta social, cultural y educativa de los países al impacto de la globalización. A través de ella el mundo académico pretende enfrentar de forma proactiva la mundialización, salvaguardando las particularidades locales.

Se puede asumir la internacionalización como "un proceso integral, que pretende incorporar la dimensión internacional e intercultural en la misión y las funciones sustantivas de las instituciones de educación superior, de tal manera que sean inseparables de su identidad y cultura. Para lograr este objetivo es preciso que las IES elaboren una política internacional explícita, adecuada a su historia, contexto y necesidades, respaldada expresamente por las autoridades universitarias, consensuada en la comunidad universitaria e implementada por una estructura administrativa y académica apropiada para su organización y promoción." (J. Gacel, 1999).

Gacel, J. (2000) reconoce que numerosos son los propósitos que justifican y respaldan los esfuerzos a favor de la internacionalización de la educación superior:

- Promover el aprendizaje de la interdependencia entre los pueblos.

- Incorporar en el proceso educativo los valores que favorecen el pluralismo, la comprensión y el respeto intercultural. Formar sujetos con las competencias requeridas para accionar con pertinencia ante un entorno cambiante.

- Incorporar a la práctica educativa el uso de las TICs.

- Lograr mayores niveles de pertinencia y calidad en las funciones y la gestión universitarias, readaptando las estructuras tradicionales.
- Articular los sistemas educativos a partir de criterios más flexibles, que faciliten la compatibilidad.

- Integrar elementos interdisciplinarios, internacionales e interculturales que generen un proceso permanente de actualización del proceso formativo.

- Promover el desarrollo institucional.

- Contribuir al desarrollo local, regional e internacional.

Madera, I. (2004) plantea que la necesidad de internacionalizar las universidades se fundamenta en tres elementos básicos:

- la colaboración entre la universidad y gremios profesionales, instituciones gubernamentales y representaciones de agencias internacionales y demás instancias que participan en este proceso. Centralización de la coordinación de la internacionalización y sus distintos procesos en una unidad al interior de la institución responsable de esta política,

- La aceptación como compromiso institucional de la participación en la construcción de una comunidad global de la educación superior.

Planificar estratégicamente, integrando la dimensión internacional requiere algo más que convertir a los centros superiores en receptores de "franquicias académicas". Esto supone (Gacel, J., 2000):

- Integrar la dimensión internacio- 
nal en la misión institucional.

- Sumar los programas internacionales en sus políticas de desarro11 .

- Asumir liderazgo y compromiso por parte de las autoridades universitarias.

- Desarrollar una cultura institucional internacional.

- Conformar un Comité de Internacionalización.

- Diseñar la política institucional de internacionalización.

- Elaborar un plan operativo de internacionalización.

- Facilitar la coordinación administrativa del proceso de internacionalización.

- Proveer el financiamiento adecuado".

De ahí, que el proceso de internacionalización universitaria en el contexto latinoamericano implica un cambio de perspectiva hacia un proceso de transformación institucional con dos componentes básicos:

- el proceso de transformación institucional (estrategias organizacionales y programáticas).

- la integración de la dimensión internacional en las funciones sustantivas (docencia, investigación y extensión), que se materializa en los resultados de pertinencia e impacto de cada uno de los procesos claves y estratégicos.

La internacionalización deberá, por tanto, favorecer aquellas actividades y acciones que se han venido creando para ello: comunicación virtual, constitución de redes, organización de asociaciones universitarias y, en los últimos tiempos, la organización de red de redes, significativos esfuerzos para no aislarse de estas formas de trabajo e integrarnos en el ámbito internacional.

Todo lo anterior posibilita a las universidades a integrar el actuar institucional con el de cada una de las áreas y dependencias que conforman la organización, permitiendo a las facultades, centros de estudio $\mathrm{u}$ otras entidades la oportunidad de gestionar y consolidar una política de proyección internacional que la transforme en un actor activo en el escenario internacional, procurando con ello, promover la integración en aquellos espacios que ofrecen oportunidades de crecimiento a partir de la promoción, el intercambio, y la cooperación tanto con sus homólogas nacionales, regionales e internacionales.

\section{II.2 Estrategia de internacionali-} zación universitaria.

La estrategia de internacionalización universitaria se orienta al desarrollo institucional universitario y se concretiza en una formulación estratégica dirigida a gestionar la dinámica del proceso de internacionalización como eje transversal de las funciones de pertinencia e impacto y gestión universitaria.

En su concepción se han empleado como métodos fundamentales el sistémico estructural-funcional y el holístico-dialéctico, los cuales permiten generalizar la propuesta principalmente para el desarrollo institucional de las universidades latinoamericanas. Para su elaboración se partió del análisis y la síntesis de la información obtenida, tanto de la bibliografía consultada, como de investigaciones realizadas en otras instituciones relacionadas con el objeto de estudio. La misma consta de tres partes: diseño estratégico, dinámica de la estrategia y metodología estratégica.

Diseño estratégico: Se establece el direccionamiento de la estrategia, desde sus propósitos, resultados esperados y descripción general. En su concepción han de ser tomados en cuenta el objetivo, así como los lineamientos estratégicos institucionales.

La estrategia de internacionalización se orienta hacia el desarrollo institucional universitario. Su direccionamiento estratégico se expresa en su propósito y en los resultados esperados; sintetizando lo institucional y lo contextual.

El propósito de la estrategia está en contribuir al desarrollo institucional universitario, de sus actores clave, funciones, procesos y relaciones, al incorporar en su cultura y su dinámica la perspectiva universal que propicie su desarrollo culturaluniversalización, desde una gestión integrada de la internacionalización como eje transversal de la praxis universitaria. Para ello han de 
realizarse acciones estratégicas que favorezcan el desarrollo de la capacidad, la voluntad y la identidad de la comunidad universitaria desde y para la internacionalización, proceso dinamizado en el marco de la colaboración-cooperación, a fin de promover el cambio e incidir en la relevancia universitaria con visión local, regional y global.

Resultados esperados: la implantación de la estrategia de internacionalización universitaria tiene con fin último un desarrollo cultural universalizado sostenido, sustentado en relaciones y valores de colaboración-cooperación, que contribuya a la relevancia institucional en la sociedad actual. Por ello, ha sido concebida para contribuir a la consecución de los siguientes resultados:

1. Redimensionamiento de la misión institucional, a partir de la integración de una visión universal en las metas y objetivos de la universidad, cuyo cumplimiento contribuya a su relevancia social en el contexto global.

2. Mayor alcance de los objetivos institucionales en el ámbito de la internacionalización, los cuales han de responder a criterios inherentes a la internacionalización para el desarrollo institucional universitario, desde sus elementos institucionales (voluntad y capacidad de la institución para el desarrollo cultural), sus elementos de vinculación procesos y actividades de internacionalización.

3. La estrategia de internacionalización se integra al sistema de gestión institucional, lo que potencia su contribución al desarrollo institucional de la universidad.

4. Desarrollar las mejores prácticas en el ámbito de la internacionalización, que puedan ser compartidas con la comunidad académica nacional e internacional y con la sociedad en general.

5. Voluntad y capacidad de la institución optimizada, desde el desarrollo de la formación y la proyección institucional universalizadas.

6. Identidad institucional más universal y auténtica, que contribuya a la visibilidad de la universidad.

7. Voluntad, capacidad e identidad para la internacionalización de la comunidad desarrollada en forma sostenida, de modo que fortalezca su compromiso con el cambio institucional y social desde una visión universal, a partir de planes, mecanismos y acciones diseñados para tales fines. Estos han de sustentarse en el desarrollo culturaluniversalización, la integración y la colaboración-cooperación.

8.Una comunidad académica más cooperadora y articulada, en virtud de una gestión de la internacionalización integradora.

La estrategia de internacionalización orientada hacia el desarrollo institucional universitario, ha sido diseñada como un sistema de procesos, articulados desde tres componentes principales, denominados mecanismos de implantación. Estos son: a) los sistemas de gestión de la internacionalización, b) los grupos focales y de reflexión y c) los centros de excelencia, los cuales convergen en interfaces de desarrollo. Los mismos integran elementos normativos, estructuralfuncionales, estratégicos, formativos, tecnológicos, entre otros, que favorecen la sinergia institucional en el ámbito de la internacionalización. Como totalidad, este sistema constituye un componente del macrosistema de gestión institucional. (figura1)

Esta estrategia presenta un carácter integrador, el cual se expresa en el diseño y funcionamiento de sus mecanismos de implantación, así como en la naturaleza de su dinámica, concebidos para facilitar la incorporación de la internacionalización en la cultura y la praxis institucional, su aplicación requiere de mecanismos de gestión que permitan su incorporación en el sistema universitario de forma integral, los cuales propicien la activación de la capacidad transformadora, la transversalidad, la sostenibilidad y el impacto que definen y sustentan dicho modelo en sus elementos esenciales.

Por tanto, la estrategia opera en tres niveles simultáneos, a partir de los cuales actúa como eje transversal y como proceso de gestión: 


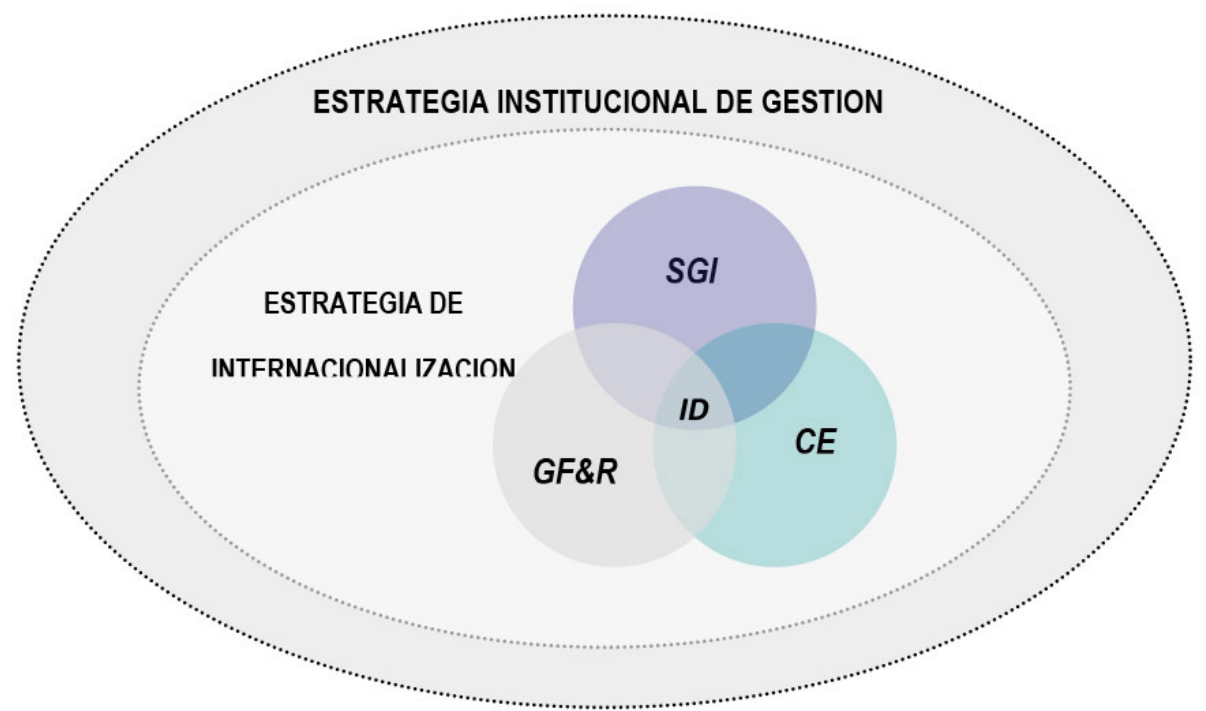

*SGI: Sistema de Gestión de la Internacionalización $\quad{ }^{*}$ CE: Centros de Excelencia

*GF\&R: Grupos Focales y de Reflexión $\quad{ }^{*}$ ID: Interfaces de desarrollo

Fuente: elaboración propia.

un primer nivel de sensibilización, un segundo nivel estratégico o de transformación y un tercer nivel de concreción práctica o desarrollo sostenido. Los dos primeros permiten la integración de la estrategia institucional de internacionalización como dimensión a su sistema. Se propone considerar las regularidades que caracterizan la internacionalización como eje transversal que impacta las políticas, objetivos estratégicos, estructuras, funciones, actores clave y relaciones. El tercer nivel definido en las líneas estratégicas de internacionalización y sus acciones programáticas, que se en- foca las regularidades inherentes a las actividades derivadas de las estrategias y acciones que se desarrollan a lo largo del proceso de internacionalización.

La estrategia de internacionalización está conformada por componentes articulados en forma sinérgica, a partir de los cuales se ha de propiciar el desarrollo de la voluntad, la capacidad y la identidad de la comunidad universitaria para la internacionalización. La activación de estos componentes (sistemas de gestión de la internacionalización, centros de excelencia y gru- pos focales y de reflexión) define el funcionamiento de la estrategia, a partir de la dinámica interna que la caracteriza.

La capacidad de ejecución a la estrategia viene dada por la puesta en marcha de sus tres mecanismos de implantación:

1. Sistema de gestión de la internacionalización (SGI) universitaria como sustento normativo, estructural-funcional y operativo de la estrategia de internacionalización orientada hacia desarrollo institucional universitario. Este sistema parte de la necesidad identificada 
de integrar la dimensión internacional en forma sistémica, a fin de potenciar su impacto en el desarrollo institucional y social. Esto ha de lograrse mediante su incorporación a los procesos de mejora continua del sistema universitario, las funciones universitarias, la modernización de la gestión y los servicios, el establecimiento de nuevos patrones de comportamiento en los actores claves institucionales, así como de relaciones internas y con el entorno, lo cual se hace viable a través de los componentes del SGI.

De aquí que se defina el SGI como el conjunto articulado de políticas, estrategias y acciones que, a través de una estructura funcional y de gestión, sustenta un proceso sinérgico e integrado de dirección y acción, que asegura la integración de la dimensión internacional en la cultura y la dinámica institucional.

El mismo establece los medios y modos de implantación y desarrollo de la estrategia de internacionalización orientada hacia el desarrollo institucional universitario, como sustento normativo, estructural-funcional y estratégico, a fin de propiciar el desarrollo.

La funcionalidad del sistema está dada por la articulación de los tres componentes esenciales, concebidos con la finalidad de materializar el desarrollo de la voluntad, la capacidad y la identidad de la comunidad universitaria para la internacionalización:
A.Política de internacionalización, cuya función es establecer los principios y lineamientos generales que fundamentan y orientan la dimensión internacional en la praxis universitaria. Se expresa en las políticas generales de la universidad, misión, visión y objetivos estratégicos institucionales, en los cuales se debe institucionalizar la dimensión internacional como elemento consustancial a su identidad. A su vez, define las políticas de internacionalización que han de establecer el marco de actuación y la declaración de principios de la política de internacionalización de la institución; así como, proveer los lineamientos generales que orienten su accionar.

B. Estructura organizacional-funcional para la gestión de la internacionalización, dirigida a diseñar y consolidar una estructura de relaciones y funciones que este modelo propone para gestionar los sistemas de internacionalización (SGI), que permita la implantación de la política institucional y el desarrollo de las estrategias, a partir de la colaboración-cooperación intra y extra sistema. Expresa la forma en que la universidad reordena sus recursos y define relaciones que permiten la implantación en forma participativa de la política de internacionalización. Su comportamiento matricial va a permitir que la dimensión internacional pueda ser asumida con funcionalidad y compromiso por todas las instancias institucionales.

C.Estrategia de gestión cuya función es planificar, dirigir, supervisar y evaluar, con sentido estratégico, las distintas acciones e iniciativas que contribuyen a integrar la dimensión internacional en los procesos universitarios, conforme a las políticas. Estas estrategias se definen en atención a la Estrategia institucional de internacionalización, los objetivos y líneas estratégicas del SIG, las acciones Programáticas que permiten la implantación de las estrategias y la evaluación de la misma.

2. Grupos focales y reflexión. $(G F \mathcal{E} R$ ) que se refiere a los espacios de diálogo, comunicación y formación, diseñados para incentivar la participación dirigida y consciente de los miembros de la comunidad académica y promover el desarrollo cultural-universalización desde la integración de pensar (lo cognitivo), el sentir (lo afectivo) y el hacer (lo psicomotor) del ser humano. Estos tienen el propósito de: a) Explorar actitudes, prioridades, valores, creencias y marcos conceptuales relativos al proceso de internacionalización orientado hacia el desarrollo institucional universitario, el desarrollo cultural-universalización, la colaboración-cooperación y aspectos vinculados a la estrategia. De esta forma, los actores clave tendrán la oportunidad de participar 
y analizar críticamente la experiencia particular e institucional en el ámbito de la internacionalización, establecer consenso y contribuir a la toma de decisiones; b) Propiciar la formación de valores y actitudes que favorezcan el desarrollo de la voluntad, la capacidad y la identidad de la comunidad universitaria para la internacionalización, mediante la conformación de pequeños grupos de estudio y reflexión, así como la utilización de otras estrategias grupales, entornos virtuales de aprendizaje y comunicación, entre otros recursos. Se recomienda la realización de talleres y plenarias diseñados para propiciar la comunicación a mayor escala y alcance.

3. Los Centros de Excelencia (CE) constituyen un mecanismo de carácter sistémico, óptimo para facilitar el desarrollo de mejores prácticas de internacionalización en las universidades. Se conciben a partir de la articulación de componentes y subcomponentes internos y externos, enfocados en la formación de la capacidad requerida para la consecución de mejores prácticas. Incluye conocimientos, habilidades, soportes y recursos tecnológicos que facilitan la sinergia interna del sistema y de éste con su entorno. Tiene como propósito articular las capacidades y voluntades institucionales, lo que incluye las redes externas de cooperación y medios de apoyo, a fin lograr el desarrollo de mejores prácticas de internacionalización para el desarrollo institucional universitario, compar- tibles con la comunidad académica nacional e internacional.

Los centros de excelencia son mecanismos de fácil implantación en las instituciones, principalmente en calidad de actividades estratégicas que permiten materializar resultados previstos en los planes de desarrollo institucional. Promueven la transformación del sistema y de su entorno, al tener como principio de actuación la puesta en común de la mejor práctica alcanzada.

4. La Interface de desarrollo (ID) se convierte en el mecanismos de integración para la implantación, se requiere a la articulación de los distintos componentes, a fin de llevar a cabo acciones específicas que tengan como resultado el desarrollo de voluntad, la capacidad y la identidad de la comunidad universitaria para la internacionalización. Las interfaces de desarrollo son espacios de intersección entre diferentes mecanismos de implantación de la estrategia, los cuales convergen para concretar y hacer viable los objetivos estratégicos desde acciones programáticas. Éstas posibilitan a los actores clave interactuar con la estrategia y la hacen visible. A través de ellas se construyen significados y sentidos que contribuirán a lograr los objetivos y alcanzar los resultados esperados.

\section{II.3 Implementación de la estratégica.}

La implantación de la estrategia de internacionalización universi- taria se fundamenta en la estrategia de gestión desarrollada por $\mathrm{H}$. Fuentes y col (2003a), La misma ha sido validada como referente en investigaciones desarrolladas y tesis defendidas exitosamente, algunas de ellas relacionadas con el objeto de esta investigación (Mellado Pérez, B., 2005; Díaz, C., 2005). Está conformada por un conjunto de procedimientos a partir de los cuales se define su direccionamiento y planeación, relacionados dialécticamente. La figura 2 muestra el sistema con sus elementos direccionamiento y planeación de la estrategia, estableciendo sus relaciones y etapas por niveles. El primer nivel permite definir el direccionamiento de la estrategia, se realiza desde la unidad con los elementos que dan soporte al direccionamiento estratégico de la institución, lo que marca su proyección. En el mismo se establece el es y el deber ser de la dimensión internacional y la estrategia de internacionalización propuesta en esta investigación, en la plataforma institucional y normativa de la universidad. Se define, además, su orientación estratégica, a partir de la armonización de las necesidades e intereses institucionales y las necesidades y tendencias del contexto.

La estrategia de internacionalización universitaria se sustenta en los valores que han sido formados desde sistema simbólico que sustenta su cultural, sus expresiones espirituales y materiales, la historia, experiencia, el contexto y demás 
elementos y que han permitido ir conformando valores universales, regionales, territoriales e institucionales que definen la cultura y la identidad de la institución y su comunidad. Las premisas y requisitos se constituyen en antecedentes de los valores institucionales y a la vez los retroalimentan.

Otra fase clave de este nivel está dada por el análisis de la misión y la visión institucional, las cuales determinan la orientación de las políticas, metas, estrategias y planes de la universidad, lo que incluye la estrategia propuesta en esta investigación. Asimismo, su impacto transformador sobre la cultura y la dinámica institucional, desde la integración de una visión universal, se ha de constituir en requerimiento imprescindible para redimensionar la misión, la visión, la planeación institucional, así como las políticas, objetivos estratégicos y operativos específicos para esta estrategia.

Para ello se requiere tanto el uso de estrategias de investigación social y educativa, como en análisis FODA, para la identificación de amenazas, oportunidades, fortalezas y debilidades, a fin de determinar las variables que inciden en la definición de los escenarios y el contexto donde se desarrolla el proceso, así como las regularidades que se dan en el objeto como un todo, en este caso la internacionalización para el desarrollo institucional universitario.
El segundo nivel, de carácter intermedio, estratégico o de transformación parte de los resultados del diagnóstico estratégico, el cual permite evaluar los factores internos y externos que inciden en el cumplimiento de la misión, relacionados con la propuesta de estrategia que se presenta. En esta fase se establecen los requerimientos o factores que determinan la aplicabilidad de la estrategia de internacionalización universitaria como resultados del estado del arte, tendencias, retos y del análisis interno: situación actual de la voluntad, la capacidad y la identidad de la comunidad universitaria para la internacionalización, las estructuras y recursos institucionales -incluye la formación y sus procesos -, los métodos de gestión, entre otros.

Esto deriva en la definición de la visión estratégica que orienta la propuesta, síntesis prospectiva que direcciona la proyección institucional en el ámbito de la internacionalización, definida desde la información diagnóstica, las regularidades y los escenarios. En este caso implica desarrollar la voluntad y la capacidad para el cambio hacia la universalización, a fin de transformación la identidad de la comunidad universitaria desde la internacionalización, sustentada en relaciones de cooperación.

Aquí, la visión estratégica se concreta en el objetivo estratégico que se ha de establecer para cada uno de los procesos definidos, sin definir jerarquías de unos sobre otros, en tanto se considera que todos son determinantes para que al desarrollar la misión se alcance la visión y que exprese el propósito fundamental del modelo, desde la estrategia.

A partir de la conceptualización de una macroestrategia de internacionalización universitaria, se establecen los objetivos estratégicos conforme a las dimensiones del modelo a ser desarrolladas. Estos objetivos concretan los criterios de medida o indicadores que permiten evaluar su cumplimiento, punto de partida para establecer las acciones, constituyendo la base de la microestrategia, la cual tiene un carácter programático desde la planeación.

Los planes de acción en los que se establecen los objetivos y las acciones programáticas, se definen tomando en cuenta las fases o etapas que dinamizan el proceso de desarrollo institucional universitario desde la estrategia propuesta: a) formación para los procesos de internacionalización, b) sistematización y gestión del proceso de internacionalización y c) impacto de la dinámica de la dimensión de internacionalización universitaria en el desarrollo cultural-universalización que propicie el desarrollo institucional y social. De igual modo, han de responder a las necesidades identificadas en los diagnósticos y análisis estratégicos. 
Figura 2. Estrategia de internacionalización universitaria.

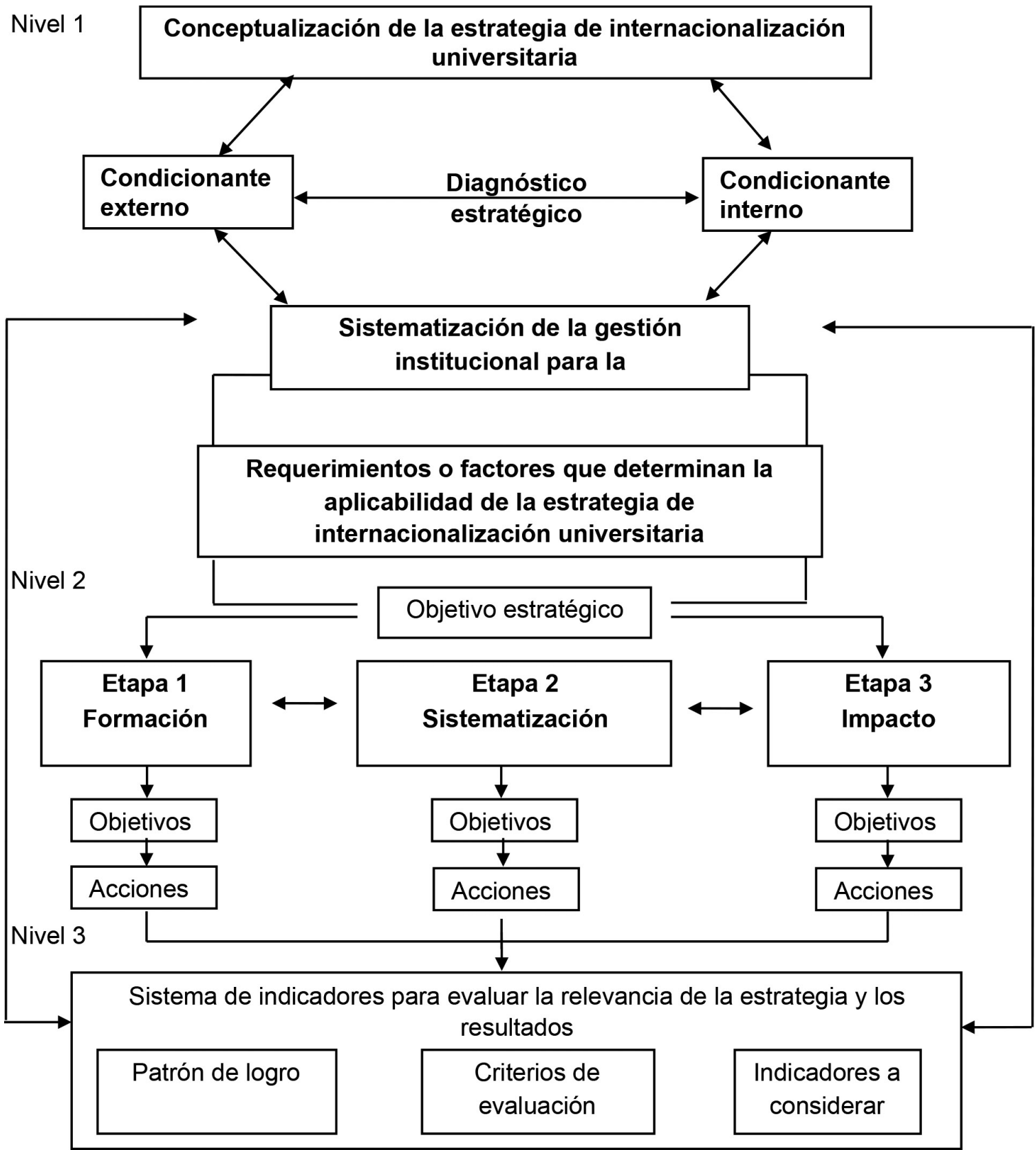

Fuente: elaboración propia. 
La singularización de esta estrategia para una institución de educación superior, se ha de llevar a cabo en correspondencia con las premisas y requisitos particulares de la institución, que prevean tanto el actual desarrollo como los escenarios futuros.

En todos los niveles y etapas de la implantación de la estrategia, deben estar presentes los elementos y resultados del modelo y estrategia de desarrollo institucional universitario, en función de la transformación y búsqueda del perfeccionamiento del proceso de internacionalización universitario. Esto requiere, además, implicar a toda la comunidad universitaria a través de su desarrollo cultural de internacionalización desde lo más universal del conocimiento.

\section{Resultados}

III.1 Consideraciones acerca de la estrategia y su implantación.

La estrategia está diseñada con el propósito de hacer potenciar el desarrollo de la cultural de la internacionalización desde la posibilidad de universalizar el conocimiento universitario para propiciar el desarrollo institucional universitario desde la internacionalización. Diversas son las investigaciones relacionadas con el proceso de internacionalización, algunas de la cuales proponen diferentes modelos, estrategias y procedimientos de internacionalización. No obstante, es criterio de estas autoras que las propuestas analizadas precisan complementarse con mecanismos propicien el desarrollo institucional desde la internacionalización. Ello plantea la necesidad de elaborar estrategias que contribuyan a desarrollar la capacidad y la voluntad de la comunidad universitaria para la internacionalización, a través de la formación y la formulación de un proyecto institucional transformador que integre lo universal en la identidad universitaria.

La presente propuesta ha permitido, definir objetivos y estrategias que, desde una praxis, se materializan en sus respectivos planes de acción, criterios de medida y grados de consecución. Estos atienden a elementos que determinan y connotan la relevancia universitaria desde la perspectiva internacional, entendida como eje trasversal que dinamiza el desarrollo institucional de las funciones de pertinencia e impacto y de gestión de la institución.

Con ello se pretende contribuir a superar las limitaciones que, desde la internacionalización, limitan la relevancia institucional universitaria en la sociedad actual, situación que ha quedado evidenciada en estudios internacionales, regionales y locales, así como en el diagnóstico realizado en esta investigación. La propuesta aporta, además, una estrategia de internacionalización orientada hacia el desarrollo institucional de la universidad, que tiene como base el modelo de desarrollo cultural - universalización para la internacionalización universitaria.

La formulación, gestión y control de los objetivos estratégicos, así como de las acciones, se desarrollan de forma integrada y en un proceso de retroalimentación permanente que se complementa a partir de la conformación de una propuesta de seguimiento, evaluación y control, permitiendo a la Universidad perfeccionar la propuesta de desarrollo institucional desde la internacionalización y establecer un sistema de trabajo en el cual participan no solo los actores implicados directamente con la internacionalización, sino toda la comunidad universitaria, como sujetos transformadores y activos dentro de la estrategia.

\section{Conclusiones}

En la fundamentación teórica de la estrategia de internacionalización universitaria potencia el desarrollo institucional universitario permitiendo revelar que la internacionalización de la universidad en la sociedad actual, se constituye en célula dinamizadora del proceso. En su desarrollo, potencia la transformación de la universidad y por ende de la sociedad.

Se reconoce a la internacionalización como un proceso multidimensional dirigido al desarrollo de la visión, las capacidades y las estrategias que requiere la universidad para accionar con relevancia en el contexto local, regional y global. 
La implantación de la estrategia en las universidades se realiza desde el reconocimiento diagnóstico de las características y condiciones reales en que se aplica, a través de las políticas, las estructuras y los planes estratégicos, encauzándose las acciones que permitan transformar la comunidad universitaria conforme a las necesidades actuales y de futuro.

La estrategia opera desde el desarrollo de la voluntad, la capacidad y la identidad a partir de los tres mecanismos de funcionamiento: sistema de gestión de la internacionalización, grupos focales y de reflexión y centros de excelencia. Su aplicación parte de la consideración de las especificidades de la institución y prevé las acciones acorde a las regularidades, el contexto y las políticas institucionales.

Se evidencia que el desarrollo institucional universitario se ve redimensionado desde una perspectiva localista a una visión universal de la educación superior, sus actores y contextos, cuando se conciben e implantan interfases que articulan los mecanismos desde acciones significativas y desarrolladoras. Ello permite la participación colectiva en la construcción de una praxis institucional internacionalizada a todos los niveles. De este modo se propicia que la universidad, desde su propia gestión y esencia, se proyecte, se desarrolle y amplíe los ámbitos de su visión y práctica internacional y con ellos de desarro- llo cultural- universalización y su capacidad de cooperación.

\section{Referencias:}

Alonso J. (2003). Principales Instrumentos de la Cooperación Internacional al Desarrollo: Factores de Cambio. [en-línea]: www.gestiopolis.com/la-cooperacion-internacional-para-el-desarrollo/

Altbach, P. (2000). Perspectives on International Higher Education. (Formatoelectrónico)

Altbach,P; J. Knight (2007). The Internationalization of Higher Education: Motivations and Realities. Journal of Studies in International Education, Vol. 11, No. 3-4, 290-305 DOI: $10.1177 / 1028315307303542$

Andrade, G. (s/f). Gestión del Cambio y de Conocimiento en las Universidades. (Formato electróni$\mathrm{co})$.

ANUIES (2002). Calidad e Internacionalización en la educación. [en-línea] http: / / www.anuies.mx (1999) Cooperación, movilidad estudiantil e intercambio académico. México. [en-línea] http: / / www.anuies.mx/servicios/destrategicos/documentos_ estrategicos/

Aponte, C. y otros (2002). Guía para la Internacionalización de las Instituciones de Educación Superior de Colombia. Editorial Secretaria General, Colombia
Aragón, L. (2000). Internacionalización de la Educación Superior: Desafíos y Oportunidades en América Latina. (Paper)

Asociación Dominicana de Rectores de Universidades, ADRU (2006). Sistema de Indicadores de Calidad de la Educación Superior Dominicana. Santo Domingo. República Dominicana

Ayala, C. (2002). La cooperación Internacional en la Educación Superior. Estudio de caso. El programa de Intercambio y movilidad (PIMA) de la OEI. Tesis en opción al grado de Master en Cooperación Internacional Unión Europea-Americana Latina. Instituto de Investigaciones Dr. JoseMaria Luís Mora. Universidad Rey Juan Carlos.

Barnett, R. (2002). Claves para entender la universidad en una era de supercomplejidad. En: Colección Educación y Conocimiento. Ediciones Pomares, S. A. Girona. [en-línea] http://www.estudiosinstitucionales-uamc.org/desin/ pdf-seminario2007/ proyecto-mfr. pdf (Consulta: abril 2006)

Bernuy, L. (2006). Tarea pendiente. Retos y posibilidades de la alianza Universidad-cooperación internacional en proyectos de impacto social. Editorial Línea Andina. Lima.

Camarena, T. (2004). Internacionalización de la Educación Superior en la República Dominicana. 
Santo Domingo. ESALC-UNESCO. (2006). Situación y

Perspectiva de la Internacionalización en la Educación Superior. Foro Presidencial por la Excelencia de la Educación. Santo Domingo.

CINDA (1992). Manual de Gestión de la Cooperación Internacional. Santiago de Chile.

(1998). Gestión de la docencia e internacionalización en las universidades chilenas. Santiago de Chile.

Crespo, I. (2005) Proyecto “Una Agenda para la Cooperación en material de Educación Superior entre España y América Latina" Instituto Universitario de Investigación Ortega y Gassett. Madrid.

De Wit, H.; J. Knight (1999).Quality and Internationalisation in Higher Education.OECD.

De Wit H. (1998). Rationales for Internationalisation of Higher Education. [en-línea] http:/ / www.ipv. $\mathrm{pt} /$ millenium/wit11.htm

(2002). Internationa-

lization of Higher Education in the United States of America and Europe: A Historical, Comparative, and Conceptual Analysis. Westport, CT: GreenwoodPress.

De Wit, H., I. Jaramillo, J. GacelÁvila, J. Knight,, editores, (2005). Educación Superior en América Latina. La dimensión Internacional. Banco Mundial_Mayol Ediciones,
Nietzsche (la filosofía). [en-línea] http: / / www.con-versiones.com / textos/nota0434.doc.

Delors J. (1996). La Educación Encierra un Tesoro. UNESCO. París. [en-línea]: www.unesco.org/education/pdf/DELORS_S.PDF

Didou, S. (2005). Informe Internacionalización y Proveedores Externos de Educación Superior en los países de América Latina y el Caribe: Principales Problemáticas. Caracas IESALC, UNESCO. [en-línea] www.iesalc.unesco.org.ve

Didriksson, A. (2000). La Universidad de la Innovación. Una estrategia de transformación para la construcción de universidades del futuro. Zacatecas- México UNESCO.

Fuentes H. et. al. (2004). La Teoría Holístico - Configuracional su consideración en los procesos sociales, CeeS “M. F. Gran”. Universidad de Oriente.

Fuentes H, Matos E. (2005). La Teoría Holístico - Configuracional y su Aplicación en los Procesos Sociales. En: Revista de la Universidad de Los Andes. Tachira. Venezuela.

Fuentes H, Matos E, Montoya J. (2006). La Teoría Holístico - Configuracional: una alternativa epistemológica en la construcción del conocimiento científico. CeeS. "M. F. Gran". Universidad de Oriente. Universidad de los Andes. Táchira. Venezuela.
Gacel-Ávila J. (2000). Internacionalización de la Educación Superior en América Latina y el Caribe. Reflexiones y Lineamientos. México.

(2003). Internacionalización de la Educación Superior: Paradigma para la Ciudadanía Global. México.

Gacel-Ávila, J., M. Diderich, p. Laus. P. (2005) Guía de Buenas Practicas en la Cooperación Universitaria entre Latinoamérica y Europa.

Green.M., C. Olson. (2003) Internationalizing the Campus A User s Guide. A. C. E. Washington, DC.

Green M; B. Hill; C. Olson. (2005). Building a Strategic Framework for Comprehensive Internationalization. A.C.E. Washington, D.C.

IESALC-SEESCyT (2003). Diagnósticos de la Educación Superior Dominicana (1-8).

IAU (2005). InternationalizationSurvay. [en-línea] www.unesco. org/iau

Jaramillo, I. (2002). La internacionalización de la educación superior. Red Colombiana para la Internacionalización de la Educación Superior. Presentación. Bucaramanga (Formato electrónico).

Knight J. (2001). Internacionalización de la Educación Superior. Calidad e Internacionalización de la Educación Superior. México. Bi- 
blioteca de la Educación Superior, ANUIES.

(2002). Comerciali-

zación de Servicios de Educación Superior: Implicaciones del GATS. TheObservatoryonBorderlessHigherEducation. [en-línea] http:// www.estudios-institucionales-uamc.org/pdf/paraleer1617 (Consulta: mayo2006)

Madera, Luz Inmaculada (2005). Un Nuevo Paradigma Educativo: La Internacionalización del Currículum en la Era Global. Ponencia presentada en el Cuarto Encuentro Nacional de Educación y Pensamiento. Santo Domingo. [en-línea] http: / / ofdp_rd.tripod.com/encuentro4/apec.html
(2006).

Un sistema de gestión de la Internacionalización y la Cooperación en la Universidad APEC, como eje transversal de la dinámica institucional. Publicado en el CD memorias del $\mathrm{V}$ Congreso Internacional de Educación Superior, Universidad 2006. La Habana.

ONU, OCDE, FMI, BM. (2000). Un Mundo Mejor Para Todos. Objetivos del Milenio.

Red Colombiana para la Internacionalización de la Educación Superior-RCI Nodo Bogotá (2006). El papel de la Educación Superior Colombiana ante la Internacionalización. Bogotá.
RedISTIC. (2003). Otro lado de la brecha. Perspectivas Latinoamericanas y del Caribe ante la CMSI. En Red sobre el Impacto Social de las Tecnologías de la Información y Comunicación.

Sebastián, J. (2000). La Internacionalización como instrumento para el desarrollo institucional de las universidades. Consejo Superior de Investigaciones Científicas. Madrid. (Formato electrónico).

(2006). Estrategias

y procesos en la Internacionalización de las universidades. Consejo Superior de Investigaciones Científicas Madrid. [en-línea] http// www.ascun.org.co/ eventos/ evento12/ documentos/Jsebastian1.pdf 